\title{
On Functional Connectivity and Symptom Relief After Gut-directed Hypnotherapy in Irritable Bowel Syndrome: A Preliminary Study
}

TO THE EDITOR: Irritable bowel syndrome (IBS) is a common functional gastrointestinal disorder characterized by chronic abdominal pain and alterations in bowel habits. In addition, IBS is frequently comorbid with chronic pain and psychological conditions such as depression and anxiety. Due to unknown and likely heterogeneous disease mechanisms, IBS therapy focuses mostly on symptom management. Several nonpharmacological therapies, including gut-directed hypnotherapy, have been shown to relieve pain and reduce IBS symptoms. ${ }^{1}$ Gut-directed hypnotherapy utilizes visualization exercises as well as mental relaxation techniques with the goal of modulating attention, perception, and emotional reactivity, and thereby extinguishing maladaptive classical conditioning to pain in order to regain normal bowel function. However, the mental mechanisms underlying the therapeutic effect remain unclear.

To elucidate the mental mechanisms of hypnotherapy, we previously investigated brain function in response to rectal distensions before and after gut-directed hypnotherapy or educational intervention. Patients in both treatment groups reported symptom reduction after therapy and successful treatment was associated with reduced activation in the anterior insula, an area where several studies have shown increased activation in IBS during rectal distensions. ${ }^{2}$ In the current preliminary study, we aimed to investigate if the relief of symptoms resulting from gut-directed hypnotherapy is associated with changes in anterior insula function in the absence of painful stimuli.

Therefore, we collected resting state functional magnetic resonance imaging (fMRI) data on a $1.5 \mathrm{~T}$ scanner during 10 minutes from 27 female IBS patients before and after treatment: hypnotherapy $(\mathrm{n}=16)$ and education $(\mathrm{n}=11)$. Details in patient recruitment are described in Lowén et al. ${ }^{2}$ IBS symptoms were assessed with the IBS severity scoring system in connection with fMRI. Hypnotherapy was performed by an experienced hypnotherapist using a written gut-directed script as applied in clinical settings. Participants in the hypnotherapy group attended seven 1-hour long sessions of individual hypnotherapy, approximately 1 session per week. Between sessions, the participants were instructed to practice daily, using a pre-recorded compact disc. Participants in the educational group attended 7 individual teaching sessions of 45 minutes each. Functional connectivity between bilateral anterior insula and the whole brain was assessed using the CONN functional connectivity toolbox ${ }^{3}$ and results were corrected for multiple comparisons using false discovery rates (FDR).

The data show that there were no differences in connectivity between the 2 groups before treatment. After gut-directed hypnotherapy, functional connectivity decreased between the right anterior insula and the right inferior parietal lobe, $\mathrm{p}_{\mathrm{FDR}}<0.001$ (Fig. 1A), and bilateral superior parietal lobes, $\mathrm{p}_{\mathrm{FDR}}<0.001$ (left) and $\mathrm{p}_{\mathrm{FDR}}$ $=0.009$ (right) (Fig. 1A and 1B). IBS symptom relief correlated significantly with connectivity decreases between the right anterior insula and both the right inferior parietal lobe, $r=0.55$, and bilateral superior parietal lobes, $r=0.48$ (left) and $r=0.48$ (right). Although there were trends of post-treatment connectivity decrease in the education group (Fig. 2), there was no such correlation between connectivity decrease and improved symptoms.

In the current study, we found evidence for the first time that in the absence of induced visceral pain, but through the use of resting state data, the anterior insula_ — associated with the abnormal central processing of pain in IBS — showed reduced connectivity following gut-directed hypnotherapy. Particularly, we found that IBS symptom reduction correlated with connectivity decreases to the right inferior parietal lobe which has been associated with attention to the spatial location of pain, ${ }^{4,5}$ perception, and attentional reorienting ${ }^{6,7}$ and also to the subjective interpretation of bodily states. ${ }^{8-10}$ Thus, our data support the general hypothesis that hypnotherapy modulates attention, perception, and subjective awareness of aversive feelings by decoupling maladaptive classical conditioning to pain and thereby reducing symptoms. 

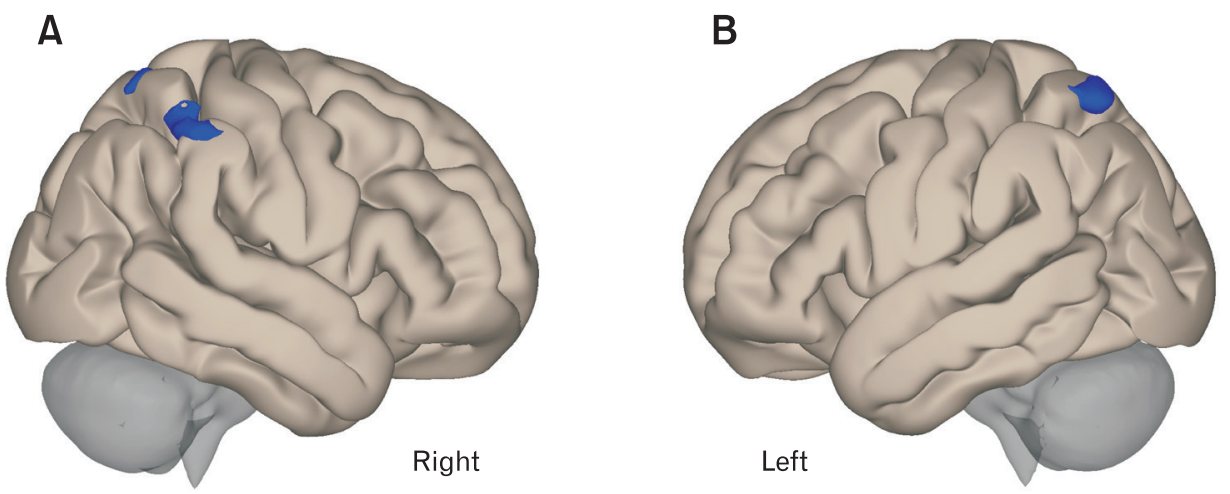

Figure 1. Post-treatment anterior insula connectivity changes. (A, B) The wholebrain connectivity between the right anterior insula and the right inferior parietal lobe and the bilateral superior parietal lobe decreased after hypnotherapy.

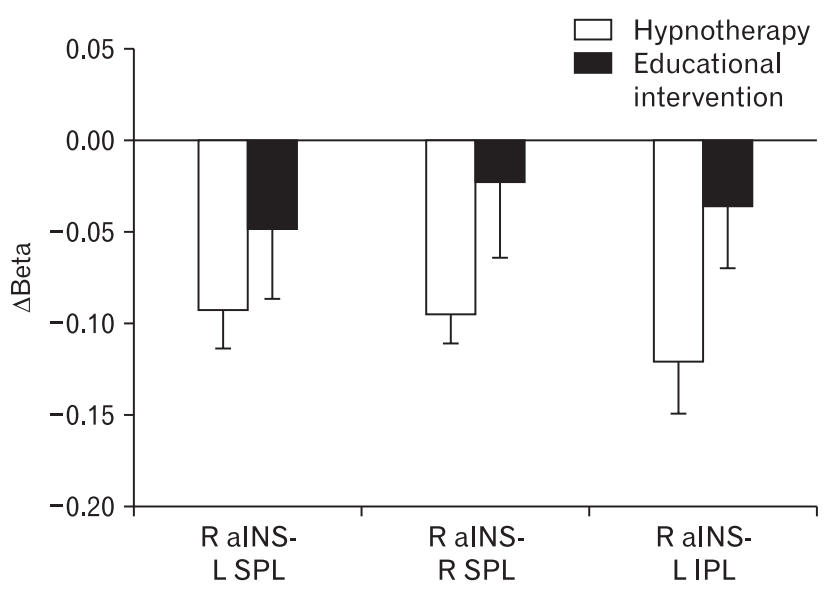

Figure 2. Functional connectivity changes ( $\Delta$ Beta) in both the gutdirected hypnotherapy and educational intervention groups. Posttreatment connectivity changes in the hypnotherapy group were significant according to the whole-brain analysis $\left(\mathrm{p}_{\mathrm{FDR}}<0.05\right)$, whereas the connectivity changes in the educational group were not significant. aINS, anterior insula; SPL, superior parietal lobe; IPL, inferior parietal lobe; R, right; L, left.

\section{Rozalyn A Simon, ${ }^{1,2 *}$ Maria Engström, ${ }^{1,2}$ Adriane Icenhour, ${ }^{3,4}$ Mats Lowén, ${ }^{2,3}$ Magnus Ström, ${ }^{3}$ Kirsten Tillisch, ${ }^{5}$ Emeran Mayer, ${ }^{5}$ Sigrid Elsenbruch, ${ }^{3,4}$ and Susanna Walter ${ }^{2,3}$}

${ }^{1}$ Department of Medical and Health Sciences, Linköping University, Linköping, Sweden; ${ }^{2}$ Center for Medical Image Science and Visualization (CMIV), Linköping University, Linköping, Sweden; ${ }^{3}$ Department of Clinical and Experimental Medicine, Linköping University, Linköping, Sweden; ${ }^{4}$ Institute of Medical Psychology and Behavioral Immunobiology, University Hospital Essen, University of Duisburg-

Essen, Essen, Germany; and ${ }^{5}$ G. Oppenheimer Center for Neurobiology of Stress and Resilience, Vatche and Tamar Manoukian Division of Digestive Diseases, David Geffen School of Medicine at UCLA, USA

1. Wilson S, Maddison T, Roberts L, Greenfield S, Singh S; Birmingham IBS Research Goup. Systematic review: the effectiveness of hypnotherapy in the management of irritable bowel syndrome. Aliment Pharm Ther 2006;24:769-780.

2. Lowén MB, Mayer EA, Sjöberg M, et al. Effect of hypnotherapy and educational intervention on brain response to visceral stimulus in the ir- ritable bowel syndrome. Aliment Pharm Ther 2013;37:1184-1197.

3. Whitfield-Gabrieli S, Nieto-Castanon A. Conn: A functional connectivity toolbox for correlated and anticorrelated brain networks. Brain Connect 2012;2:125-141.

4. Kulkarni B, Bentley DE, Elliott R, et al. Attention to pain localization and unpleasantness discriminates the functions of the medial and lateral pain systems. Eur J Neurosci 2005;21:3133-3142.

5. Peyron R, Laurent B, Garcia-Larrea L. Functional imaging of brain responses to pain. A review and meta-analysis (2000). Neurophysiol Clin 2000;30:263-288.

6. Igelström KM, Graziano MSA. The inferior parietal lobule and temporoparietal junction: a network perspective. Neuropsychologia 2017;105:70-83.

7. Igelström KM, Webb TW, Kelly YT, Graziano MS. Topographical organization of attentional, social, and memory processes in the human temporoparietal cortex. eNeuro Published Online First: 29 Apr 2016. doi: 10.1523/ENEURO.0060-16.2016.

8. Blanke O, Slater M, Serino A. Behavioral, neural, and computational principles of bodily self-consciousness. Neuron 2015;88:145-166.

9. Geng JJ, Vossel S. Re-evaluating the role of TPJ in attentional control: contextual updating? Neurosci Biobehav Rev 2013;37:2608-2620.

10. Tsakiris M, Costantini M, Haggard P. The role of the right temporoparietal junction in maintaining a coherent sense of one's body. Neuropsychologia 2008;46:3014-3018.

Acknowledgements: Marta Sjöberg at Ersta Hospital, Karolinska Institutet, Stockholm, Sweden is acknowledged for performing the gut-directed hypnotherapy and for valuable discussions regarding the study.

Financial support: This study was provided in part by Region Östergötland and the Bengt-Ihre Fond.

Conflicts of interest: None.

Author contributions: Rozalyn A Simon and Maria Engström contributed equally to the work. Rozalyn A Simon and Maria Engström wrote and analyzed the data; Adriane Icenhour edited and contributed to conversations in analysis; Mats Lowén and Magnus Ström collected data and recruited patients; and Magnus Ström, Kirsten Tillisch, Emeran Mayer, Sigrid Elsenbruch, and Susanna Walter contributed to study design and editing.

ClinicalTrials.gov Identifier: NCT01815164. 